\title{
Computation on the Optimal Control of Networked Control Systems with Multiple Switching Modes Over High Speed Local Area Networks
}

\author{
Gang Zheng, Wenli Zeng, Fanjiang Xu
}

\begin{abstract}
The optimal control problem for the networked control system with multiple switching modes over high speed local area networks is addressed, where an initial state is a parametric vector. Because in the general case, the time delay is much less than the sampling period and the possibility of the packets collision is much lower, it can be assumed that the influence of the time delay and the packets loss on the optimal controller design can be ignored. On the basis of the assumption, the networked control systems with multiple switching modes are modeled as a hybrid system. Moreover, based on the Bellman type inequality for the hybrid systems, a dynamic program to solve the optimal control with a parameter vector is proposed, in every step of the technique, the feasible region is divided into evenly distributed grid points, and then, the optimal control law is transformed into maximizing the lower bound of the cost to go function in grid points. Finally, an experiment setup of the networked control system with multiple switching modes is constructed and a simulation example is given to illustrate the optimal control computation results.
\end{abstract}

Keywords: networked control systems, hybrid systems, optimal control, dynamic program, multiparametric program

\section{Introduction}

Networked control systems (NCS) are feedback control systems wherein the control loops are closed through a real-time network[1]. The defining feature of an NCS is that information (reference input, plant output, control input, etc.) is exchanged using a network among control system components (sensors, controller, actuators, etc.). The use of a communication network offers advantages in terms of reliability, enhanced resource utilization, reduced wiring, and reconfigurability. Networked control systems typically involve switching between several different modes, depending on the range of operation. In order to improve the performance of the system and save the operation cost, it is important to design an optimal control law for the systems with different switching modes. At present the methodologies of designing the optimal controllers for such networked control systems are few. [2] assumes that the controller-plant communication in NCS is periodic, the optimal controller design problem is formulated as one for sampled-data feedback systems with periodic discrete-time components, and give a necessary and sufficient condition for existence of discrete time periodic controller in terms of LMIs, and derive a controller construction algorithm. [3]discusses the optimal $\mathrm{H}_{\infty}$ control problem for networked systems with limited communication constraint, which is formulated as a periodic control problem, and proposes a heuristic search approach in conjunction with the convex optimization of controller to solve the optimization problem. The switching process in [2] and [3] is time-controlled. However, in practice, the switching may be completely determined by the system state, for such networked control systems, the whole state space is divided into cells which each correspond to a particular control mode, so that each cell has its own continuous dynamics associated to it. This type of the system is state-controlled and can be regarded as a hybrid system. Hybrid systems have attracted considerable attention in recent years [4]. Also, in practice, the initial state of networked control systems may be not determined, and for those with multiple switching modes, the unpredicted initial states may make the optimization process more complex. This paper model the class of networked control systems with multiple switching modes as a hybrid system, based on the model, a general cost function is defined and the optimal control problem 
for a general cost function is addressed, where a initial state is a parametric vector and is not predicted. Furthermore, a solution to the optimal feedback control law is proposed, which combine the dynamic program and multiparametric program. Finally, an example is given to illustrate the optimal computation results.

\section{Problem Formulation}

\subsection{Modeling networked control systems with multiple switching modes}

Consider a class of networked control systems shown in figure 1 . The plant, the sensors, the controller and the actuators are spatially distributed and connected together through a control network. In general, there are multiple control modes in the controller of the class of systems, and every mode corresponds to the range of the continuous state of the physical plant. We assume that the control network is a high speed local area network, e.g. Ethernet, and the transmitting data policy is scheduled releasing policy, the time delay is much less than the sampling period in practice and the possibility of message collision is much lower, therefore, in this paper, it is reasonable to ignore the influence of the time delay and packets loss on the optimal controller design in the networked control systems with multiple switching modes.

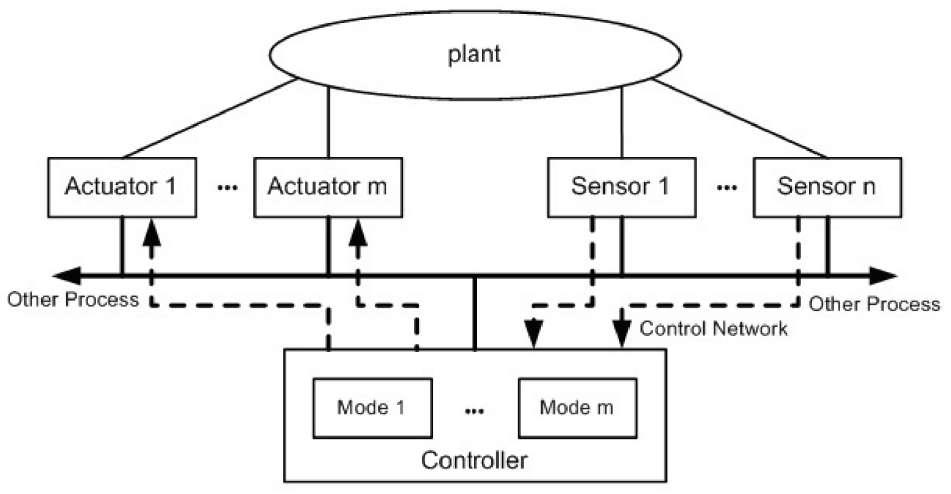

Figure 1: The networked control system with multiple control modes

On the basis of the assumption, the class of networked control systems with multiple modes can be modeled as a hybrid system. Hybrid systems contain continuous dynamics and discrete events [4]. The controller in networked control systems can calculate the state of the plant and determine the control modes, and the switching between different modes corresponds to the occurrence of the discrete event in hybrid systems. We assume that clock-driven sensors sample the plant outputs periodically at sampling instants, then the networked control system can be modeled as a hybrid system as following:

$$
\left\{\begin{array}{l}
x(k+1)=f_{q(k)}(x(k), u(k)) \\
q(k+1)=v(x(k), q(k))
\end{array}\right.
$$

where continuous state variable $x \in X \triangleq \mathbb{R}^{n}$, continuous input variable $u \in U \triangleq \mathbb{R}^{m}, k$ denotes $k$-th sampling period, $k=0,1,2, \ldots, q \in Q, Q$ is a finite countable set, whose elements correspond to the modes in the networked control system, $f_{q}: X \times U \rightarrow X$ denotes the continuous dynamics in discrete mode $q, v: X \times Q \rightarrow Q$ denotes the discrete dynamics, which is a transition process between operation modes. Without the loss of the generality, we think the transition between two modes occurs in the sampling time instant. 
The hybrid system model (1) of the networked control system with multiple modes can also be denoted a hybrid automaton[5]. In addition, if the continuous dynamics can be denoted by the linear time invariant system, the model can transformed into the piecewise affine system[6], where different modes correspond to different ranges of state variable $x$.

\subsection{Optimal Control Formulation}

Based on the model (1), the optimal control formulation in finite time horizon is discussed as follows. Firstly, define the cost function of the networked control system

$$
J\left(U_{N}, x(0)\right)=F_{q(N)}\left(x_{N}\right)+\sum_{k=0}^{N-1}\left[F_{q(k)}\left(x_{k}, u_{k}\right)+G\left(x_{k}, q(k), q(k+1)\right)\right]
$$

where $N$ is the time horizon, starting from the state $x_{0}=x(0), x_{k}$ denotes the continuous state vector at time $k$ and $q(k)$ denotes the discrete state vector corresponding operation mode at time $k$, the column vector $U_{N} \triangleq\left[u_{0}^{T}, \ldots, u_{N-1}^{T}\right]^{T} \in \mathbb{R}^{m}, F_{q(N)}\left(x_{N}\right)>0$ is a cost at end time, $F_{q(k)}\left(x_{k}, u_{k}\right)>0$ is a cost for continuous evolution in an operation mode and $G\left(x_{k}, q(k), q(k+1)\right) \geq 0$ is a cost for switching from one mode to another mode, if there is no switching, i.e. $q(k)=q(k+1), G\left(x_{k}, q(k), q(k+1)\right)=0$. Note that, we don't consider the problem of infinitely many jumps in transition time instants. Also, it should be noted that $q(0)$ is not written in the left of cost function (2), because the operation modes correspond to the range of the state variable, if the initial condition $x(0)$ is given, and the discrete mode $q(0)$ is fixed, too.

The optimal control problem of the networked control system is

$$
\begin{gathered}
J^{*}(x(0)) \triangleq \min _{\left\{U_{N}\right\}} J\left(U_{N}, x(0)\right) \\
\text { s.t. }\left\{\begin{array}{l}
x_{k+1}=f_{q(k)}\left(x_{k}, u_{k}\right) \\
q(k+1)=v\left(x_{k}, q(k)\right) \\
x_{N} \in X_{f} \\
x_{0}=x(0)
\end{array}\right.
\end{gathered}
$$

where $X_{f}$ is the terminal region, in addition, $X_{j}$ denotes the set of states $x_{j}$ at time $j$ for which (3) is feasible.

In general, the optimal control problem (3)-(4) may not have a minimum for some feasible $x(0)$. This is caused by discontinuity of the hybrid system in the input space. We will consider problem (3)-(4) assuming that a minimizer $U_{N}^{*}(x(0))$ exists for all feasible $x(0)$.

Note that in this paper we will distinguish between the current state $x(k)$ of the system (1) at time $k$ and the variable $x_{k}$ in the optimization problem (3)-(4), that is the predicted state of the system (1) at time $k$ obtained by starting from the state $x_{0}=x(0)$ and applying the input sequence $u_{0}, \ldots, u_{k-1}$ to system (1) - Analogously, $u(k)$ is the input applied to system (1) at time $k$ while $u_{k}$ is the $k$-th optimization variable of the optimal control problem (3)-(4).

In the optimal control problem (3)-(4), the initial state $x(0)$ is a vector of parameters, the goal in this paper is to solve (3)-(4) for all values of $x(0)$ of interest, and to make this dependence explicit. Therefore, the optimal control problem of the networked control system with multiple modes is a multiparameter program problem.

\section{Solution to the Optimal Control of the Networked Control Systems}

For the networked control systems with multiple modes, the optimal control problem can be viewed as a parametric programming one. The parametric program is one of the important optimization techniques in mathematical program, where the optimality is affected by the uncertainty of the parameters 
in the cost function or the constrained conditions. Because the parameters are either unknown or that will be decided later, parametric programming need to subdivide the space of parameters into characteristic regions, which depict the feasibility and corresponding performance as a function of the uncertain parameters, and hence provide the decision maker with a complete map of various outcomes [6].

In the networked control system, different operation modes correspond to different range of the state variable, the switching sequence between different modes is related with the initial condition of the system, which is not fixed. If the switching sequence is fixed, the optimal control problem is equivalent to a finite time optimal control problem for a hybrid system, which can be solved by multiparametric program, in this case, the optimal solution is a optimal control candidate for the networked control system. If all of the switching sequences in the networked control system are given, the solution to the optimal control problem (5) can be obtained by comparing the values of cost function corresponding to the optimal control candidate in different switching sequences.

However, with the increase of the control modes, the computing process gets more complex and the computing efficiency gets much lower because we must enumerate all the possible switching sequences of the networked control system, which is a little same as the famous traveling salesman problem (TSP) in the optimization theory. In order to decrease the computation complexity and improve the computation efficiency, the dynamic program technique is proposed to solve the optimal control problem of the networked control system with multiple control modes.

The dynamic programming solution of the optimal control problem is denoted as follows:

$$
\begin{gathered}
J_{n}^{*}\left(x_{n}\right) \triangleq \min _{\left\{u_{n}\right\}}\left\{\left[F_{q(n)}(x(n), u(n))+G(x(n), q(n), q(n+1))\right]+J_{n+1}^{*}(x(n+1))\right\} \\
\text { s.t. } x(n+1) \in X_{n+1}, n=N-1, \ldots, 0
\end{gathered}
$$

with terminal conditions:

$$
\begin{aligned}
\chi_{n} & =X_{f} \\
J_{N}^{*}(x(N)) & =F_{q(N)}\left(x_{N}\right)
\end{aligned}
$$

where $X_{j}$ is the set of all initial states for which dynamic program problem (5)-(8) is feasible, $X_{j}=\{x \in$ $\left.\mathbb{R}^{n} \mid \exists u, f_{q(j)}(x, u) \in X_{j+1}\right\}$.

The first step of dynamic program is as follows:

$$
\begin{aligned}
& J_{N-1}^{*}(x(N-1)) \triangleq \min _{\left\{u_{N-1}\right\}}\left\{\left[F_{q(N-1)}(x(N-1), u(N-1))+G(x(N-1), q(N-1), q(N))\right]\right. \\
&\left.+J_{N}^{*}(x(N))\right\} \\
& \text { s.t. }\left\{\begin{array}{l}
f_{q(N-1)}\left(x(N-1), u_{N-1}\right) \in X_{f} \\
J_{N}^{*}(x(N))=F_{q(N)}\left(x_{N}\right)=F_{q(N)}\left(f_{q(N-1)}\left(x(N-1), u_{N-1}\right)\right)
\end{array}\right.
\end{aligned}
$$

It is clear that the optimal control problem (9)-(10) is a multiprarametric program problem, where $x(N-1)$ is a parametric vector. In terms of terminal region $X_{f}$ and the system equation (1), the feasible region $X_{N-1}$ at time $N-1$ is obtained. [7] discuss the discretized computation on the optimal control of hybrid systems, where the initial state and the terminal state are determined and the key point is to search the maximum lower bound of the value function. The parameter vector increases the complexity of computing the optimal control feedback law. Based on the algorithm in [7], the value function $V_{q}(x)$ is introduced, when $x=x_{0}, q=q_{0}, V_{q_{0}}\left(x_{0}\right)$ is a lower bound on the cost for optimally bring to the system from determined initial state $\left(x_{0}, q_{0}\right)$ to the terminal state $\left(x_{t_{f}}, q_{t_{f}}\right)$. It should be noted that without the loss of the generality, we discuss the case where continuous state space is two-dimensional system. 
Let $e_{1}=\left[\begin{array}{ll}1 & 0\end{array}\right]^{T}, e_{2}=\left[\begin{array}{ll}0 & 1\end{array}\right]^{T}, \forall x \in X_{N-1}$, let

$$
\begin{aligned}
& x_{N-1}^{j k}=x_{N-1}+j h e_{1}+k h e_{2}, \\
& x_{N-1}^{j k} \in X_{N-1}, \text { and } j, k \in \mathbb{Z}, \\
& Y_{N-1}^{j k} \triangleq\left\{x: x=x_{N-1}^{j k}+\theta_{1} h e_{1}+\theta_{2} h e_{2},-1 \leq \theta_{1} \leq 1,-1 \leq \theta_{2} \leq 1\right\}, \\
& \left(\underline{f}_{q(N-1)}^{j k}\right)_{i}=\min _{x \in Y_{N-1}^{j k}, u \in U}\left\{f_{q(N-1)}(x, u) e_{i}\right\}, \\
& \underline{F}_{q(N-1)}^{j k}=\min _{x \in Y_{N-1}^{j k}, u \in U}\left\{F_{q(N-1)}(x, u)\right\}, i=1,2 \\
& V_{q(N-1)}^{j k}=V_{q(N-1)}\left(x_{N-1}^{j k}\right), \\
& \Delta_{i} V_{q(N-1)}^{j k}=\left(V_{q(N-1)}\left(x_{N-1}^{j k}+h e_{i}\right)-V_{q(N-1)}\left(x_{N-1}^{j k}\right)\right) / h, \\
& \Delta_{-i} V_{q(N-1)}^{j k}=\left(V_{q(N-1)}\left(x_{N-1}^{j k}\right)-V_{q(N-1)}\left(x_{N-1}^{j k}-h e_{i}\right)\right) / h, \quad i=1,2 .
\end{aligned}
$$
ities:

Introduce new vector variables, $\lambda_{q(N-1)}^{j k} \in \mathbb{R}^{2}$ for $(j, k)$ at time $N-1$, construct the following inequal-

$$
\left\{\begin{array}{l}
0 \leq\left(\lambda_{q(N-1)}^{j k}\right)_{1}+\left(\lambda_{q(N-1)}^{j k}\right)_{2}+\underline{F}_{q(N-1)}^{j k} \\
\left(\lambda_{q(N-1)}^{j k}\right)_{|i|} \leq\left(\underline{f}_{q(N-1)}^{j k}\right)_{|i|} \Delta_{i} V_{q(N-1)}^{j k}, i=-2,-1,1,2 \\
\left(\lambda_{q(N-1)}^{j k}\right)_{|i|} \leq\left(\bar{f}_{q(N-1)}^{j k}\right)_{|i|} \Delta_{i} V_{q(N-1)}^{j k} \\
0 \leq V_{q(N)}^{j k}-V_{q(N-1)}^{j k}+G\left(x_{N-1}^{j k}, q(N-1), q(N)\right), x_{N-1}^{j k} \in S_{q(N-1), q(N)} \\
0 \geq V_{q(N)}^{x_{N}}, x_{N} \in X_{f}
\end{array}\right.
$$

If there exists $V_{q(N-1)}^{j k}$ such that inequalities (11) hold, which are Bellman type inequalities. It can be proved that that $V_{q(N-1)}^{j k}$ is the lower bound of the cost to go function $J_{N-1}\left(x_{N-1}\right)$, and the proof is given in [7]. However, any function that meets the constraints is a lower bound on the cost to go function, thus to yield the useful bounds, it is necessary to find an maximum of all grid points in the feasible region $x_{N-1}$,

$$
J^{*}(x(N-1)) \triangleq \max _{x_{N-1}^{j k} \in X_{N-1}, j, k \in \mathrm{Z}}\left\{V_{q(N-1)}^{j k}\left(x_{N-1}^{j k}\right)\right\}
$$

Assuming that

$$
V_{q(N-1)}^{j_{0} k_{0}}=\max _{x_{N-1}^{j k} \in X_{N-1}, j, k \in \mathrm{Z}}\left\{V_{q(N-1)}^{j k}\left(x_{N-1}^{j k}\right)\right\}
$$


when $j=j_{0}, k=k_{0}$, for $x=x_{j_{0} k_{0}}+\theta_{1} h e_{1}+\theta_{2} h e_{2} \in Y_{N-1}^{j_{0} k_{0}}$, define the interpolating function:

$$
\begin{aligned}
& V_{q(N-1)}(x)=\left(1-\theta_{1}\right)\left(1-\theta_{2}\right) V_{q(N-1)}^{j_{0} k_{0}}+\theta_{1}\left(1-\theta_{2}\right) V_{q(N-1)}^{\left(j_{0}+1\right) k_{0}} \\
& +\left(1-\theta_{1}\right) \theta_{2} V_{q(N-1)}^{j_{0}\left(k_{0}+1\right)}+\theta_{1} \theta_{2} V_{q(N-1)}^{\left(j_{0}+1\right)\left(k_{0}+1\right)}
\end{aligned}
$$

From (14), the optimal feedback control law can be calculated as

$$
u_{N-1}=\underset{u \in U}{\arg \min }\left\{\frac{\partial V_{q(N-1)}}{\partial x} f_{q(N-1)}(x, u)+F_{q(N-1)}(x, u)\right\}
$$

so far, the optimal control law at the first step is finished.

From the second step $n=N-2$ to the last one $n=0$, the cost to go function is defined on the feasible region $X_{n+1}$, we will solve the problem (5), which is still a multiparametric program problem, the computing process is analogous as the first step, which is presented as follows:

(I) Divide the feasible region $X_{n}$ into evenly distributed grid points, and introduce $x_{n}^{j k}, Y_{n}^{j k},\left(\underline{f}_{q(n)}^{j k}\right)$, $\left(\bar{f}_{q(n)}^{j k}\right), \underline{F}_{q(n)}^{j k}, V_{q(n)}^{j k}, \Delta_{i} V_{q(n)}^{j k}, \Delta_{-i} V_{q(n)}^{j k}, i=1,2$;

(II) Solve the linear program problem:

$$
\begin{aligned}
& J^{*}(x(n)) \triangleq \max _{x_{n}^{j k} \in X_{n}, j, k \in \mathrm{Z}}\left\{V_{q(n)}^{j k}\left(x_{n}^{j k}\right)\right\} \\
& \text { s.t. }\left\{\begin{array}{l}
0 \leq\left(\lambda_{q(n)}^{j k}\right)_{1}+\left(\lambda_{q(n)}^{j k}\right)_{2}+F_{q(n)}^{j k} \\
\left(\lambda_{q(n)}^{j k}\right)_{|i|} \leq\left(\underline{f}_{q(n)}^{j k}\right)_{|i|} \Delta_{i} V_{q(n)}^{j k}, \quad i=-2,-1,1,2 \\
\left(\lambda_{q(n)}^{j k}\right)_{|i|} \leq\left(\bar{f}_{q(n)}^{j k}\right)_{|i|} \Delta_{i} V_{q(n)}^{j k}, \quad i=-2,-1,1,2 \\
0 \leq V_{q(n+1)}^{j k}-V_{q(n)}^{j k}+G\left(x_{n}^{j k}, q(n), q(n+1)\right), \quad x_{n}^{j k} \in S_{q(n), q(n+1)}
\end{array}\right.
\end{aligned}
$$

(III) After solving the maximum value of $V_{q(n)}^{j k}$, define the interpolating function $V_{q(n)}(x)$, then calculating the optimal feedback input

$$
u_{n}=\underset{u \in U}{\arg \min }\left\{\frac{\partial V_{q(n)}}{\partial x} f_{q(n)}(x, u)+F_{q(n)}(x, u)\right\}
$$

It should be noted that the dynamic program methodology to compute the optimal control feed back input in this paper is different from [6]. In [6], the cost function is quadratic and the optimal controller design is for the piecewise affine (PWA) system, if the hybrid system can not be modeled as a PWA system, and the methodology in computing the optimal controller needs to be further discussed. Also, in [7], the initial state and the terminal state are determined, thus there are no parameters in the optimal control problems. In this paper, the initial state is a vector parametric vector, the solution to optimal control must make an dynamic enumeration to search the maximum lower bound in every sample period, the problem is different from [7].

\section{Simulation Example}

We use the local area network in the laboratory to construct a networked control system, where the network contains Ethernet and the communication between nodes is done using TCP/IP sockets. The experiment setup is shown in figure 2 . 


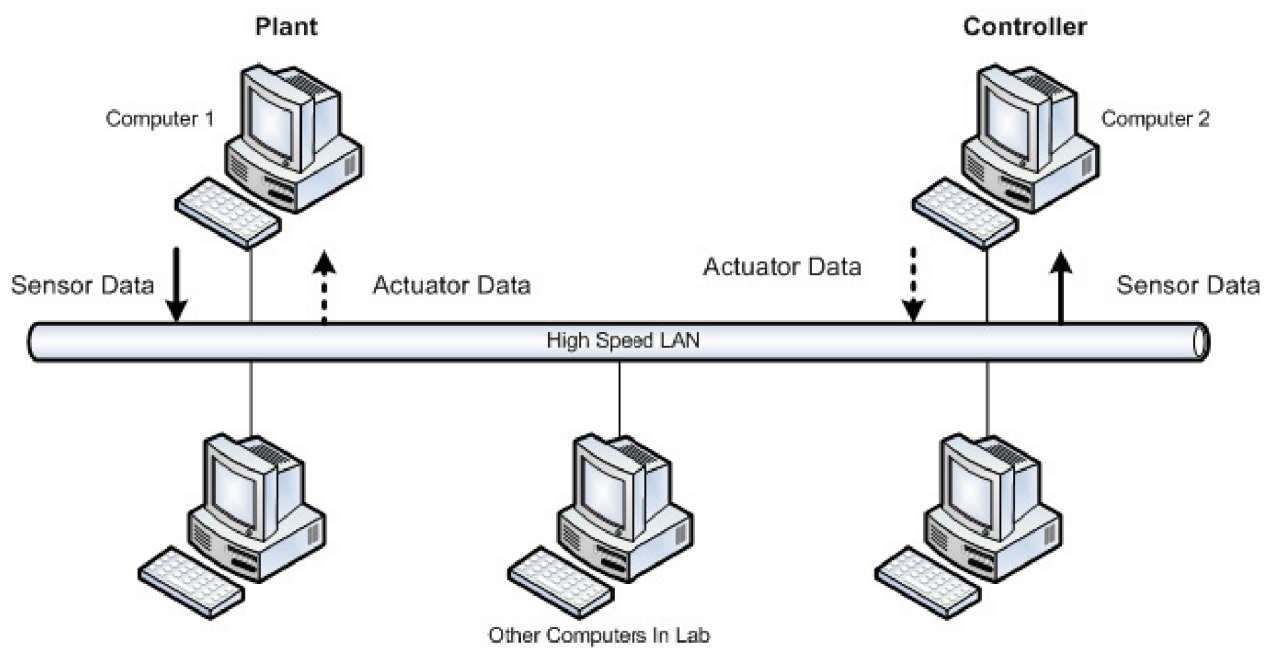

Figure 2: The networked control system experiment setup

In our experiment setup, one computer (Computer 1) works as a plant, and the other computer (Computer 2) works as a controller, these two computers and the other computers are connected over the local area net in the lab. Computer 1 simulate the dynamics of the plant, which can obtain the control signal from the net, make the output calculation, and send the computation results to the controller computer. Computer 2 obtains the plant output, and calculates the control signal, then send the control signals to the plant computer. Note that the networked control systems with multiple switching modes can be carried out in the experiment setup. Based on the hybrid system model of the networked control system, the different dynamics of the plant can be simulated in the plant computer, and the controller computer calculates the control results and sends them to the plant computer and control the dynamics of the plant in different modes.

Because TCP/IP is used in the Ethernet in the lab, the end to end data transmission is reliable, and the packets loss between the plant computer and the controller computer can not be considered. But the network-induced delay needs to be considered when the plant computer and the controller computer exchange data across the network. We test the delays between two computers in the network, it can be found that the time delay generally maintains a fixed value, in our experiment setup, the fixed value is much less than the sampling period. Therefore, it is reasonable that the networked-induced delay can not be considered in this paper, and the model and the solution to the optimal control can be validated in this simulation.

The networked control system simulated in the experiment setup is given as follows.

$$
x(k+1)=A_{q_{i}} x(k)+B u(k), i=1,2
$$

where

$$
\begin{aligned}
& A_{q_{1}}=\left[\begin{array}{cc}
\frac{\sqrt{2}}{2} & -\frac{\sqrt{2}}{2} \\
\frac{\sqrt{2}}{2} & \frac{\sqrt{2}}{2}
\end{array}\right], A_{q_{2}}=\left[\begin{array}{cc}
\frac{\sqrt{2}}{2} & \frac{\sqrt{2}}{2} \\
-\frac{\sqrt{2}}{2} & \frac{\sqrt{2}}{2}
\end{array}\right], B=\left[\begin{array}{l}
0 \\
1
\end{array}\right], \\
& S_{q_{1}, q_{2}}=\left\{x:\left[\begin{array}{ll}
1 & 0
\end{array}\right] x(k)<0\right\}, \\
& S_{q_{1}, q_{2}}=\left\{x:\left[\begin{array}{ll}
1 & 0
\end{array}\right] x(k) \geq 0\right\}, \\
& x(k) \in[-10,10] \times[-10,10], \\
& u(k) \in[-1,1],
\end{aligned}
$$


define the cost function

$$
J\left(U_{N}, x(0)\right) \triangleq x_{N}^{T} P x_{N}+\sum_{k=0}^{N-1} x_{k}^{T} Q x_{k}+u_{k}^{T} R u_{k}
$$

where

$$
P=Q=\left[\begin{array}{cc}
700 & 0 \\
0 & 700
\end{array}\right], R=1
$$

Terminal region

$$
\begin{aligned}
& x_{f}=[-0.01,0.01] \times[-0.01,0.01], \\
& N=3 .
\end{aligned}
$$

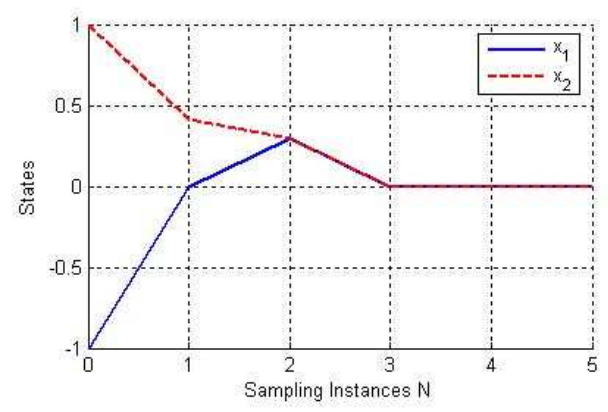

Figure 3: The optimal trajectory of the state variable $x$

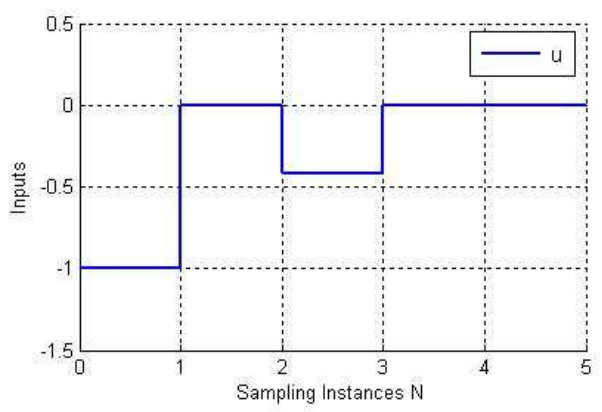

Figure 4: The optimal control input when $x=\left[\begin{array}{ll}-1 & 1\end{array}\right]^{T}$

The optimal control problem is minimizing the cost function and solving the optimal control feedback input. In terms of the steps presented in section 3 and some toolbox related with multiparametric programming [8] and optimal control [9], the solving program is designed, and then the optimal control can be obtained. When $x_{0}=\left[\begin{array}{ll}-1 & 1\end{array}\right]^{T}$, the optimal trajectory of the state variable $x$ is shown in Fig. 3, and the control input is shown in Fig. 4.

\section{Conclusion}

In networked control systems with multiple switching modes, it is necessary to calculate the optimal control feedback input, which can improve the system performance and save the cost. This paper models 
the class of networked control systems as a hybrid system and addresses a optimal control problem, where initial state is a parametric vector. Consider switching modes and unknown initial states, the dynamic program techniques are proposed to solve the optimal control problem, where every step calculation need enumerate the grid points in feasible region to find a maximum lower bound of the cost function and solve the optimal feedback input. Finally, an example is given to illustrate the techniques. It should be noted that, the switching modes in networked control systems make the calculation of the optimal control input more complex, dynamic program can reduce the calculation complexity than the heuristic enumeration. In the future research, it is an important task to improve the computation efficiency and reduce the complexity. In addition, time delays are common in networked control systems, it is an interesting area to work into the optimal control problem with delay.

\section{References}

[1] W. Zhang, M. S. Branicky , S. M. Phillips . Stability of networked control systems. IEEE Control Systems Magazine, Vol. 21, pp. 84-99, 2001.

[2] H. Fujika, K. Ito. Performance optimization for a class of networked control systems with communication constraints. Proceedings of the American Control Conference, pp. 248-253, 2003.

[3] L. Lu, L. Xie, M. Fu. Optimal control of networked systems with limited communication: a combined heuristic and convex optimization approach. Proc. of 42th IEEE Int. Conf. on CDC, pp. 119411992003.

[4] A. van der Schaft, H. Schumacher,An Introduction to Hybrid Dynamical Systems,Berlin: SpringerVerlag, 2000.

[5] R. Alur,C. Courcoubetis, T. A. Henzinger. Hybrid automata: an algorithmic approach to the specification and verification of hybrid systems. In: Grossman R L, Nerode A, Ravn A P, et al, eds. Hybrid Systems, Lecture Notes in Computer Science,Berlin: Springer-Verlag, Vol. 736, pp. 209-229, 1993.

[6] F. Borrelli, Constrained optimal control of linear and hybrid systems, Berlin: Springer-Verlag, 2003.

[7] S. Hedlund, A. Rantzer. Optimal Control of Hybrid Systems. Proc. 38th IEEE Int. Conf. on CDC,pp. 3972-3977, 1999.

[8] M. Kvasnica, P. Grieder, M. Baotic, et al, Multi-Parametric Toolbox. ETH, Swiss Federal Institute of Technology, 2004.

[9] S. Hedlund, A. Rantzer, CDP Tool: A Matlab tool for optimal control of hybrid systems. Department of Automatic Control, Lund Institute of Technology, 1999.

Authors: Gang Zheng, Wenli Zeng, Fanjiang Xu Laboratory of Integrated Information Systems Technology Institute of Software, Chinese Academy of Sciences No.4 South Fourth Street, Zhongguancun, Beijing, China, 100080 E-mail: gangzhengcn@yahoo.com.cn 